www.jmscr.igmpublication.org

Impact Factor 3.79

Index Copernicus Value: 5.88

ISSN (e)-2347-176x ISSN (p) 2455-0450

crossref DOI: http://dx.doi.org/10.18535/jmscr/v3i12.06

Journal Of Medical Science And Clinical Research

\title{
Primary Anterior Saggital Anoplasty in Female Anorectal malformation
}

\author{
Authors \\ Nitin Goel $^{1}$, Dhiraj Parihar ${ }^{2}$, Preeti Raikwar ${ }^{3}$, Arvinder Pal Singh Batra ${ }^{4}$, \\ Jeewandeep Kaur ${ }^{5}$ \\ ${ }^{1}$ Associate Professor, Pediatric Surgery, BPS GMC for Women, Sonepat \\ ${ }^{2}$ Assistant Professor, Pediatric Surgery, BPS GMC for Women, Sonepat \\ ${ }^{3}$ Associate Professor, Pediatric, BPS GMC for Women, Sonepat \\ ${ }^{4}$ Professor \& Head, Anatomy, BPS GMC for Women, Sonepat \\ ${ }^{5}$ Assistant Professor, Physiology (C R L), BPS GMC for Women, Sonepat
}

\begin{abstract}
Aim: To describe the technique and results of anterior saggital approach i.e. Anterior Saggital Anorectoplasty (ASARP) for low \& intermediate Anorectal malformation ARM

Methods: Patients with low \& intermediate Anorectal malformation (ARM) was taken for ASARP without colostomy as definitive surgery at presentation. Pre-operative preparation was done with rectal washouts and clear liquid diet for 24 hours. Those patients who require abdominal mobilization does not need to change the position during the procedure in this approach .Rectum is separated from vagina under direct vision and placed in sphincter muscle complex without dividing it .

Results: ASARP was performed on 27 patients, between September 2011 to September 2015 (age range from 3 days to 9 years). Follow up ranges from 3 months to 4 years. 3 patients had wound complication in immediate post operative period that was minor wound infection \& resolved on conservative management and no patient required colostomy. Four patients presented with anal stenosis which were successfully treated by dilatation. Mucosal prolapse was seen in one patient who was self limiting. Three patients have long term follow up with constipation.

Conclusion: Anterior Saggital approach is technically easy with acceptable complication and showed good cosmetic result in female ARM

Keywords: Anorectal malformation, Anterior Saggital Anorectoplasty, Anovestibula
\end{abstract}

\section{Introduction}

ASARP was first described by Okada in 1992. This technique offer unique advantages over the posterior approach. The avoidance of the prone position and the consequent physiological alterations during anaesthesia made this approach immensely popular. The incision is anterior to the neoanus and the posterior fibers of the levator ani are left intact which makes this procedure more technically correct. This procedure is ideal for intermediate and low female anorectal anomalies. 


\section{Observation}

This is a retrospective study of case series; data was obtained from records and follow up sheets in department of pediatric surgery of BPSGMC for women Khanpur Kalan Sonepat. Patients of female anorectal malformation were admitted through outpatients or emergency. 27 cases of female ARM with 22 cases of anovestibular fistula (AVF), 1 case of rectovaginalnal fistula and 4 cases of anteroposed anus in the period September 2011 to September 2015 ( age range from 3 days to 9 years -Table-1). The diagnosis was made by clinical examination and by insertion of suitable hegars dilator into the fistula (Table-2).

Table 1 age wise distribution of patients

\begin{tabular}{|l|c|}
\hline Age of patients & No of patients \\
\hline $0-3$ month & 12 \\
\hline $3-6$ month & 5 \\
\hline $6-12$ month & 6 \\
\hline Above 12 month & 4 \\
\hline
\end{tabular}

Table 2 shows the distribution of various female ARM

\begin{tabular}{|l|c|}
\hline Anomaly & No. of cases \\
\hline Anteposed anus & 4 (Fig-1) \\
\hline Anovestibular & 22 (Fig-2) \\
\hline Rectoveginal & 1 \\
\hline
\end{tabular}

All patients had a preoperative hemogram. X-ray abdomen for spine was done. Sonography of abdomen was done for urogenital anomalies. Micturating cystouretherogram was done to detect VUR. Echocardiography were done in those patients showing clinical features of cardiac anomaly Preoperative preparation for babies less than 1 year consisted of clear liquids for 24 hours, no milk and rectal washout 4 to 6 times with maintenance intravenous fluids

The operation is performed under endotrachel anesthesia in lithotomy position. Many patients with low anomaly were operated in caudal anesthesia with sedation. Site of anus is marked with electro stimulation. A midline incision is made from the fistula to the posterior limit of proposed site of anus. The incision is deepened and all tissue in the midline are divided, keeping strictly in midline by using muscle stimulator, until posterior bowel wall is exposed in anterior part of incision. Remaining close to bowel wall, lateral dissection is done on either side; Terminal part of rectum is dissected posteriorly and laterally remaining very close to bowel wall. Separation of rectum from vagina is done at last under direct vision by fine tip electrocautery. Anteriorly one should mobilize up to peritoneal reflection.

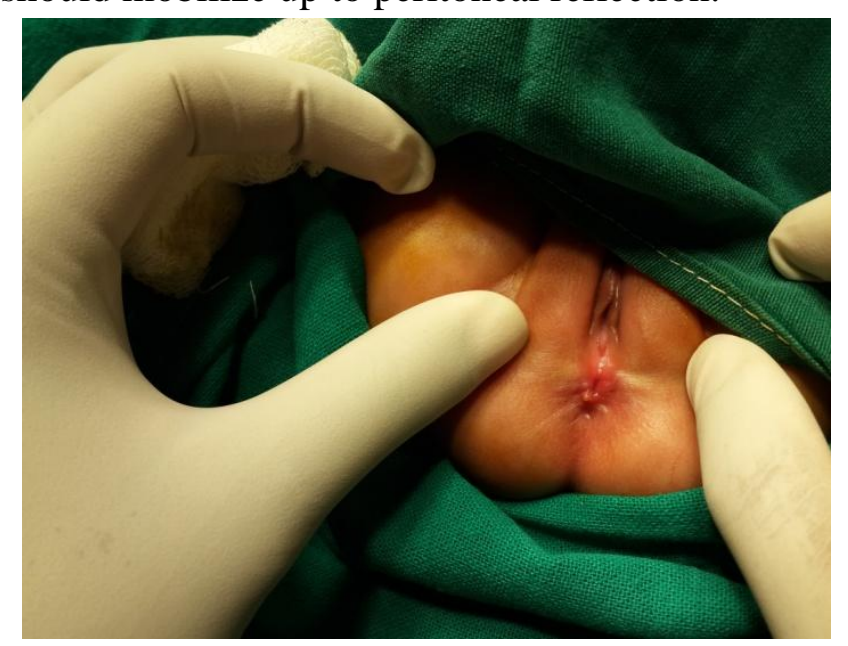

Fig 1 Illustrate Anteposed anus

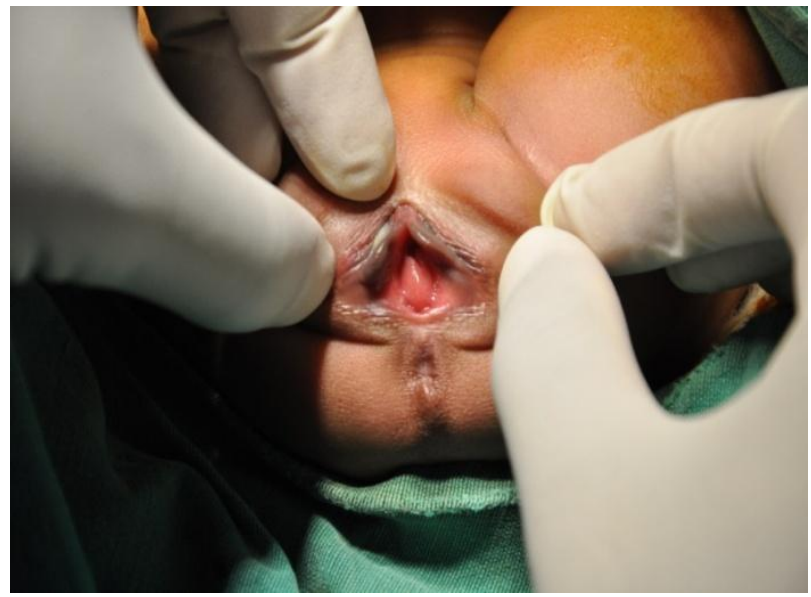

Fig 2 Illustrate Anovestibular fistula

After the rectum has been mobilized sufficiently to reach the site of anus without tension, it is placed in the center of muscle complex, which is sutured around it anteriorly and at lower part of suturing, anterior rectal wall is included .The perineal muscles are sutured anteriorly, reconstituting the perineal body. Anoplasty is performed with circumferential sutures of 4-0 vicryl. The vestibule and forchette are repaired. 
The skin is sutured creating a normal looking perineum

The patient is allowed orally next morning and no immobilization is employed. Anoplasty site is regularly cleaned by sprinkling diluted betadine solution after every stool by parents. The wound is left exposed after first 48 hours. Catheter is removed on $7^{\text {th }}$ postoperative day and patient is discharged.

Patient is followed up in outpatient department at 2 weeks and that time, we start anal dilatation as per age of patient. Oral lactulose is prescribed for 1 month postoperatively (Fig-3)

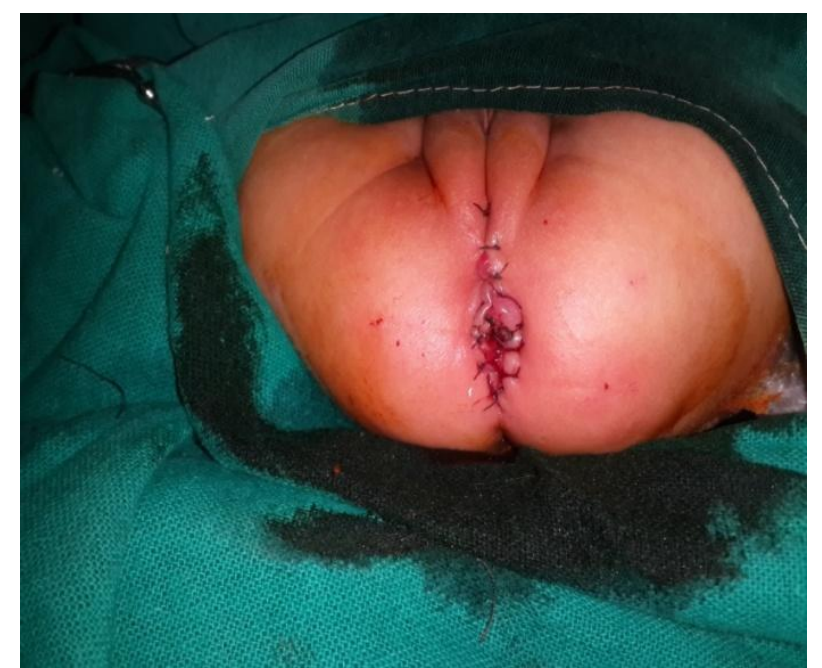

Fig 3 Post operative picture of Anterior Saggital Anorectoplasty ASARP

Follow up visit are scheduled 3 monthly for one year and then annually for 4 years. Follow up ranges from 3 months to 4 years. 3 patients had wound complication in immediate post operative period that was minor wound infection managed conservatively no one needed colostomy. Four patients presented with anal stenosis which were successfully treated by dilatation. Mucosal prolapse was seen in one patient who was self limiting. three patients have long term follow up with constipation as a major long term complication was treated by dietary management and oral lactulose .23 patients were having normal looking perineum, were fully continent and regular bowel habits .one patient with short perineal body underwent burrington's procedure .

\section{Discussion}

Surgical procedure used for treatment of low and intermediate female ARM include cut back, perineal anal transposition, Y-V plasty, colostomy followed by minimal PSARP, sacroperineal repair and ASARP. Anal transposition and PSARP are preferred approaches these procedures have been limited by incomplete anatomic exposure, blind tunneling of the rectum, lack of reconstruction of the perineal body, need for a colostomy, and a displeasing appearance of the perineum, with anterior migration of the anus in the long term. ${ }^{1}$

In 1992, Okada et al ${ }^{2}$ described a less invasive perineal approach for repair of ARMs known as the anterior Saggital anorectoplasty (ASARP). The ASARP has largely been used for the repair of rectoperineal and rectovestibular fistulas, as well as a potential reoperative approach to previously repaired ARMs. Basic aims at operation for female ARM are 1) correctly discriminate and identify sphincter muscle and to pass rectal tube through them properly, 2) adequate separation of vagina from rectum 3) adequate downward mobilization of rectum to perform a tension free anastomoses with skin.

All these aims are easily achieved in ASARP. Colostomy is avoided, the entire rectum and its local anatomy is clearly visualized during dissection. Only the anterior aspect of sphincter muscle complex is divided. Levator ani is not divided. Separation of vagina and rectum is easier due to better anatomical orientation. We feel in prone position anterior dissection is difficult. Other advantage of this approach is that it can be done under caudal anesthesia.

Okada $^{3}$ and Sanchez ${ }^{4}$ have recommended prolonged fasting and TPN; however it requires prolonged hospitalization and has poor patient tolerance and passage of hard stool which may harm suture line ${ }^{5}$. We agree with Whaklu et $\mathrm{al}^{6}$ in early starting of feeds as it prevents initial bile stools from coming in contact with the suture line resulting in wound complications. 
The result of ASARP in our series is comparable to the results published by Wakhlu et $\mathrm{al}^{6}$, Aziz et $\mathrm{al}^{7}$ with excellent stool continence and major long term complication was constipation, which was easily treatable by dietary management and lactulose. ASARP has a better cosmetic appearance than PSARP (Sherif M.K Shehata ${ }^{8}$ ).

For common cloaca Genitoscopy is done to assess the length of common channel. Most widely accepted and practiced approach consisted of a posterior Saggital operation with "total urogenital mobilization", with or without a laparotomy depending on the September 2011 to September 2015 (age range from 3 days to 9 years).complexity of the defect. This involved mobilizing the rectum off the urogenital sinus, leaving the latter structure intact without an attempt to separate the urethra from the anterior vaginal wall. The urogenital sinus is mobilized as a unit and anastomoses is performed to the anterior perineum to create a neovestibulum.

\section{Conclusion}

Anterior Saggital approach is technically easy with acceptable complication and showed good cosmetic result in female ARM. The incision is anterior to the neoanus September 2011 to September 2015 (age range from 3 days to 9 years).and the posterior fibers of the levator ani are left intact which makes this procedure more technically corrects. This procedure is ideal for intermediate and low female anorectal anomalies.

\section{Reference}

1. Wakhlu A, Pandey A, Prasad A, Kureel SN, Tandon RK. Anterior saggital Anorectoplasty for anorectal malformations and perineal trauma in female child. J Pediatr Surg, 1996; 31(9):1236-1240

2. Okada A, Tamada $\mathrm{H}$, Tsuji $\mathrm{H}$, et al. Anterior saggital anorectoplasty as a redo operation for imperforate anus. J Pediatr Surg. 1993;28: 933-8.
3. Okada A, Kamata S, Imura K, Fukuzawa M, Kubota A, Yagi M, Azuma T . Anterior saggital anorectoplasty for rectovestibular and anovestibular fistula. $\mathbf{J}$ Pediatr Surg.1992; 27(1):85-88

4. Sanchez MR, Molina E, Cerda J, Estelles C, Casillas MA, Romero R, Vazquez J .Treatment of vestibular fistulas in older girls.Cir Pediatr.2002; 15(4):140-144

5. Kulshrestha S, Kulshrestha M, Singh B, Sarkar B, Chandra M, Gangopadhyay an Anterior saggital anorectoplasty for anovestibular fistula. Pediatr Surg Int.2007; 23:1191-1197

6. Ashish Wakhlu, Shiv Narain Kureel, Raj Kumar Tandon, Avtar Kishan Wakhlu; long term results of anterior Saggital anorectoplasty for the treatment of vestibular fistula; journal of pediatric surgery; 2009; 44;1913-19

7. Aziz MA, Banu T, Prasad R, Khan AR. Primary anterior saggital anorectoplasty for rectovestibular fistula. Asian J Surg, 2006 29(1):22-24

8. Sherif M.K Shehata; prospective long term functional and cosmetic results of ASARP versus PSARP in treatment of intermediate anorectal malformations in girls; pediatr Surg Int; 2009:25:863-68

9. Pena A: Total urogenital mobilization: An easier way to repair cloacas. J Pediatr Surg, 1997; 32:263-268. 